\section{Sarah Bernardeth Fernández Diez ${ }^{1}$ Wilson Delgado Azañero² Víctor Calderón Ubaqui ${ }^{2}$ Helard Ventura Ponce ${ }^{2}$ Alfredo Zuiko Félix ${ }^{2}$}

\section{Cirujano Dentista}

Docente del Departamento Académico de Medicina y Cirugía Bucomaxilofacial.

Facultad de Estomatología. Universidad Peruana Cayetano Heredia

\section{Correspondencia}

Víctor Calderón Ubaqui

Av. Honorio Delgado 430 - Lima 31, Perú.

Teléfono: 381-1950, Anexo 252

e-mail: victor.calderon@upch.pe

Recibido : 4 de marzo de 2010

Aceptado : 10 de mayo de 2011

\section{Introducción}

El fibromixoma es una variante del mixoma odontogénico, llamado así por la gran cantidad de fibras colágenas que se observan en el examen histológico. La literatura lo describe dentro del mixoma odontogénico (MO), de tal manera que no es clasificada como una entidad independiente (1). La Organización Mundial de la Salud (OMS)

lo considera derivado del mesénquima y/o ectomesénquima que puede contener o no epitelio odontogénico (1).

Es un tumor localmente invasivo cuyas características radiológicas presentan patrones con grandes variaciones, puede manifestarse como una lesión radiolúcida unilocular o multilocular, con márgenes definidos o difusos (2-4), en algunos casos la imagen radiográfica de raqueta de tenis es bastante sugerente de la lesión pero ésta no debe considerarse patognomónica. La superposición de tejidos de la zona afectada puede dar lugar a una imagen que suele confundirse con otros tumores odontogénicos o no odontogénicos e

\title{
Fibromixoma odontogénico con mimetismo radiográfico de lesión ósea maligna. Reporte de caso
}

Fernández-Diez SB, Delgado-Azañero WA, Calderón-Ubaqui V, Ventura-Ponce H, Zuiko-Félix A. Fibromixoma odontogénico con mimetismo radiográfico de lesión ósea maligna. Reporte de caso. Rev Estomatol Herediana. 2011; 21(2):87-92.

RESUMEN

Se presenta un caso de fibromixoma odontogénico de la mandíbula en un paciente adolescente de 14 años de edad, cuyos cambios radiográficos sugerían osteosarcoma. El análisis y la integración de los datos clínicos con las imágenes de la tomografía axial computarizada fueron claves para establecer el diagnostico diferencial entre ambas entidades.

Palabras clave: FIBROMIXOMA / OSTEOSARCOMA / TOMOGRAFÍA COMPUTARIZADA ESPIRAL.

Odontogenic fibromixoma of the mandible mimicking malignant bone lesion. Case report

ABSTRACT

A case of odontogenic fibromixoma of the mandible in a 14-years-old patient which radiographic changes suggested osteosarcoma is reported. The analysis of clinical data together with the bone changes found in TC images was the clue to establish the differential diagnosis between both lesions.

Key words: FIBROMYXOMA / OSTEOSARCOMA / SPIRAL COMPUTED TOMOGRAPHY

incluso con lesiones óseas de carácter maligno (3-6). En este trabajo se presenta un caso de fibromixoma odontogénico con cambios radiográficos de lesión maligna. El cuadro clínico y la tomografía computarizada espiral multicorte fueron claves para plantear el diagnóstico de mixoma odontogénico diferenciándolo del osteosarcoma.

\section{Reporte de caso}

Un adolescente varón de 14 años de edad se presentó al servicio de Cirugía Oral y Maxilofacial de la Facultad de Estomatología de la Universidad Peruana Cayetano Heredia, refiriendo que desde aproximadamente tres meses había notado un aumento de volumen progresivo de la región hemifacial derecha que no le producía ninguna molestia. Debido a la ausencia de sintomatología no consultó ni recibió tratamiento pensando que se resolvería espontáneamente. La persistencia de la deformación, la aparición de dolor a la palpación y la reducción de la apertura de la boca lo conduce a buscar atención en nuestro servicio.
A la evaluación clínica se observó un aumento de volumen en la región maseterina y parotídea derecha, de consistencia firme, sin cambios de coloración ni temperatura de la piel que recubría la zona afectada. No se palpaban adenopatías (Fig. 1). Intraoralmente solo se evidenció la ausencia del primer molar inferior derecho el que fue extraído años atrás. (Fig. 2)

La radiografía panorámica y la escanografía de rama mostraron una imagen radiolúcida ubicada en ángulo, rama y cóndilo mandibular derecho, que estaba dividida por delicados tabiques radiopacos en lóculos de diferentes tamaños. Los límites de la lesión no eran claramente definidos y de la zona más posterior surgían delicadas líneas radiopacas que recordaban los rayos de sol descritos en el osteosarcoma (Figs. 3 y 4).

La tomografía espiral multicorte con secciones de $1,2 \mathrm{~mm}$, reconstruidos a $0,6 \mathrm{~mm}$, con y sin la administración de sustancia de contraste endovenoso, mostró imágenes con características sugestivas de neopla- 


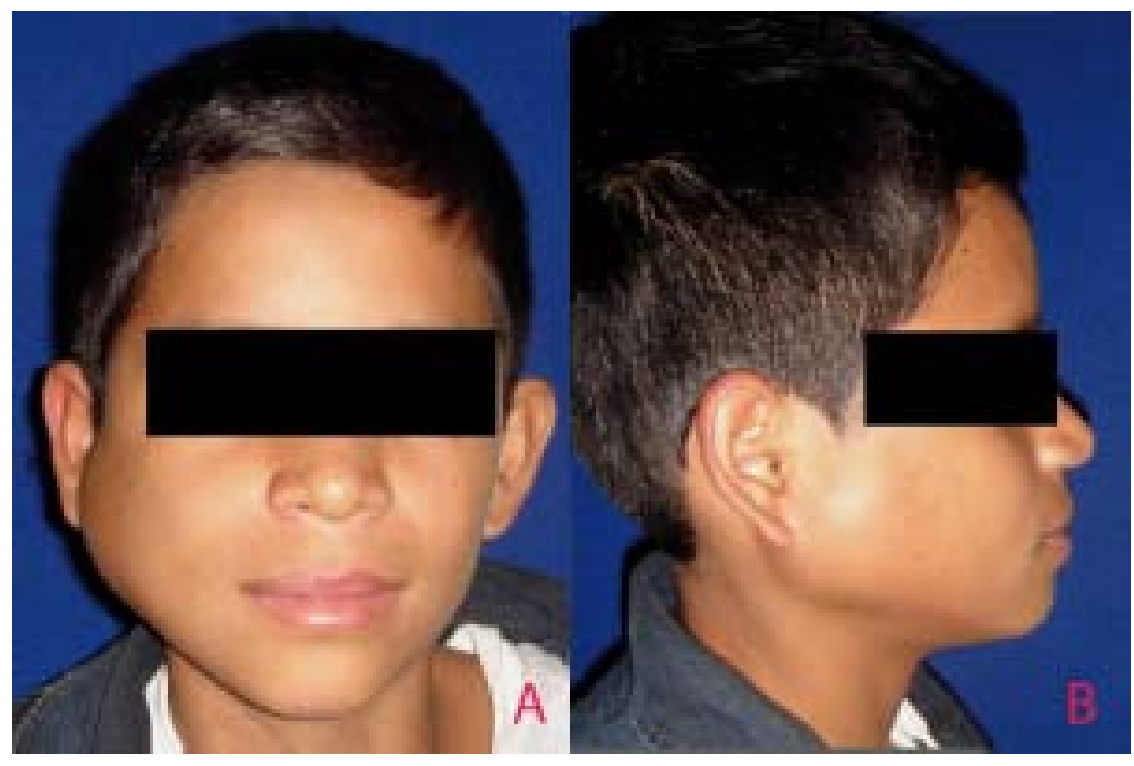

Fig. 1. A.Asimetría facial por hinchazón de la zona maseterina.B. Aumento de volumen de la zona maseterina y ángulo mandibular. No hay compromiso de glándula parótida.

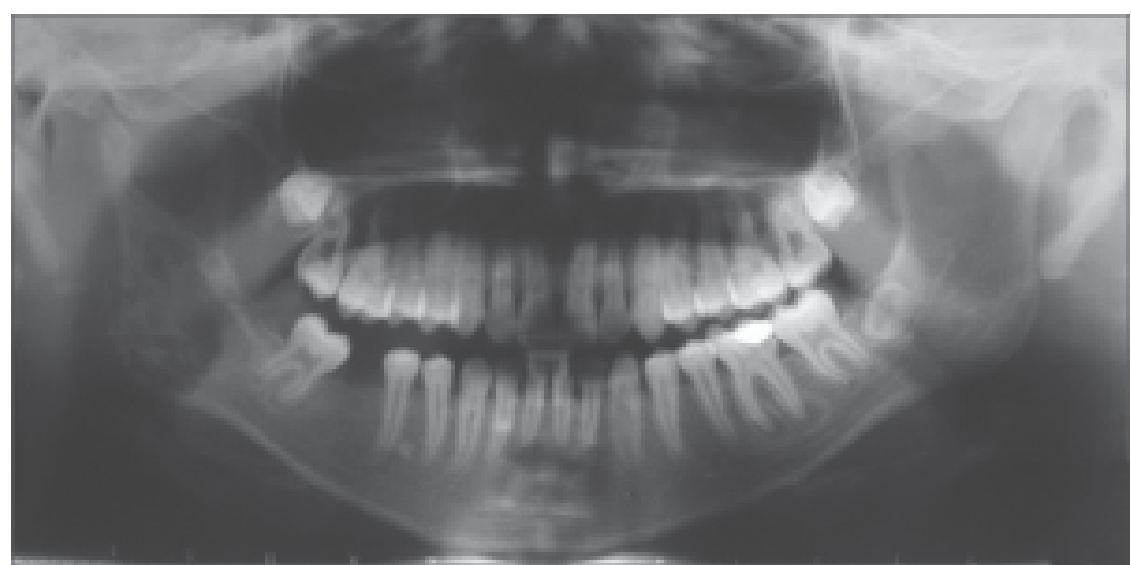

Fig. 3. La radiografía panorámica muestra extensa lesión osteolítica única en la rama ascendente mandibular derecha.

sia maligna. En los cortes axiales a nivel de la rama ascendente, se observaba una imagen hipodensa que había reemplazado la estructura trabecular ósea normal, que producía una moderada expansión y adelgazamiento de la cortical ósea vestibular con focos de perforación, limitados por septos hiperdensos que se dirigían hacia afuera dando el patrón de rayos de sol (Fig. 5), hacia lingual prácticamente no se observaba cortical ósea excepto a nivel del cóndilo donde se distinguía lóculos hipodensos limitados por tabiques hiperdensos (esclerosis) con erosión (Fig. 6).

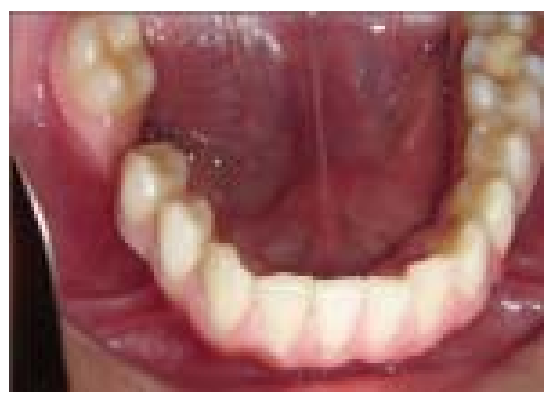

Fig. 2. Desaparición del surco vestibular sólo a nivel de la pieza 47 .

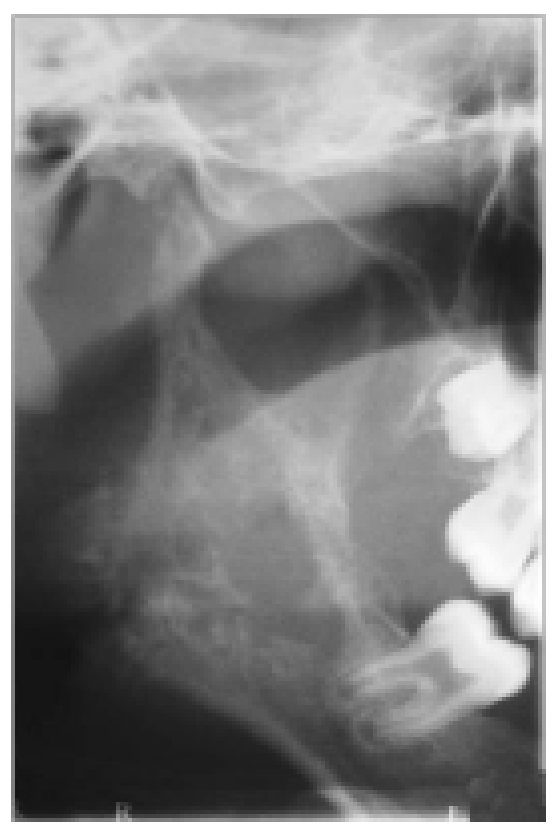

Fig. 4. Lesión radiolúcida tabicada que compromete rama, ángulo, cóndilo y apófisis coronoide de la mandíbula. Obsérvese "las radiaciones" a nivel del ángulo.

En las TAC, a pesar de la extensión de la lesión hacia las partes blandas, era posible observar los límites entre la lesión y las estructuras musculares circundantes desplazadas (interfase), aspecto que no se observa en lesiones malignas (Figs. 8 y 9), los septos internos de la lesión se extendían más allá del margen óseo, dando la apariencia de patrón de rayos de sol (Fig. 8) en forma más o menos similar a lo que se observaba en la radiografía convencional (Fig. 4). La captación de la sustancia de contraste era homogénea y no se observan adenopatías, orientando el diagnóstico hacia una 


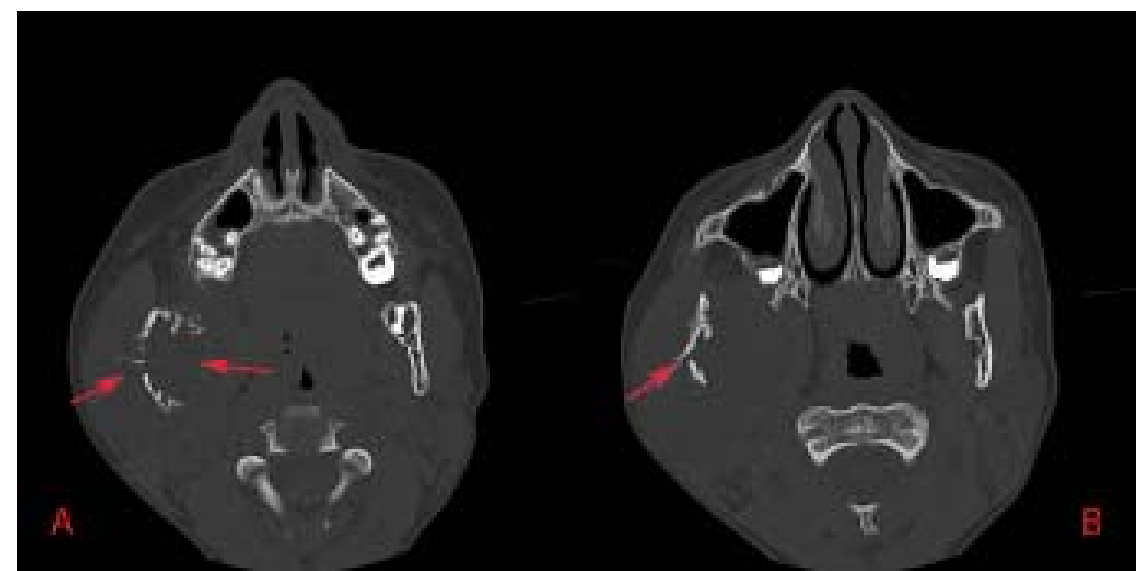

Fig. 5. TAC: imagen hipodensa con desaparición de la cortical lingual, adelgazamiento y expansión de cortical vestibular y tabiques hiperdensos que surgen de la cortical vestibular.

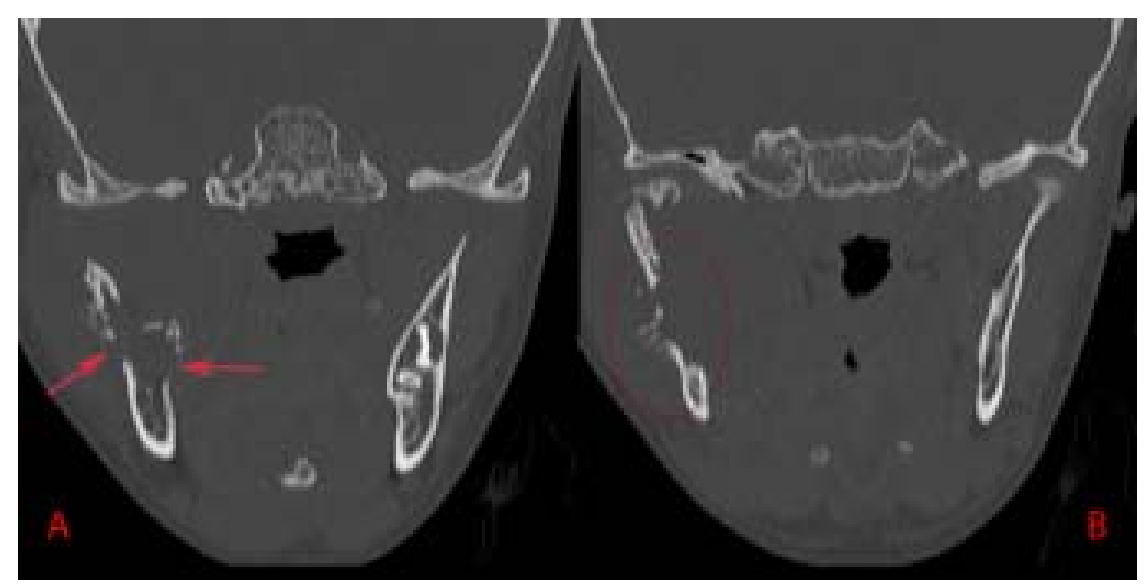

Fig. 7. A. TAC: perforación de tablas lingual y vestibular. B. "Patrón de rayos de sol" en tabla vestibular y ausencia de tabla lingual.

lesión tumoral benigna de comportamiento agresivo.

El estudio histopatológico de una biopsia incisional mostraba un tejido fibromixomatoso entremezclado con áreas de fibras colágenas distribuidas en forma desordenada. También se distinguía la presencia de algunas trabéculas óseas delgadas. No se identificaban cambios atípicos. El diagnóstico anatomopatológico preoperatorio fue fibromixoma (Fig. 10).

El tratamiento consistió en la resección parcial de la mandíbula cuya extensión abarcaba desde la cara distal de la segunda premolar y la rama ascendente incluyendo el cóndilo, en el mismo acto quirúrgico se colocó un injerto autólogo córticoesponjoso de calota craneal y de

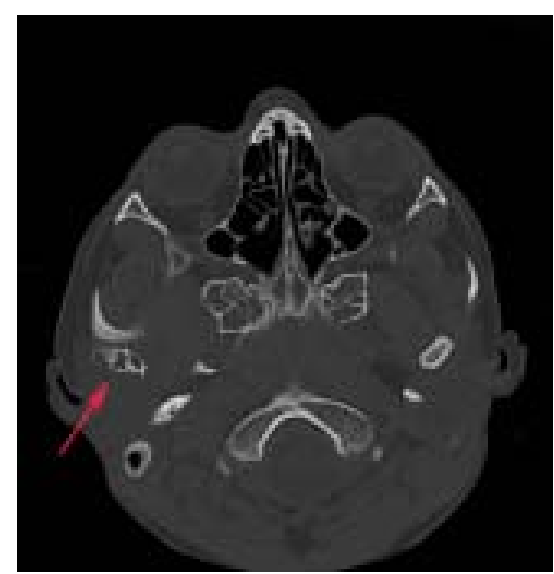

Fig. 6. TAC: cóndilo derecho con imagen hipodensa multilocular (fecha).

dilección de género, sin embargo algunos reportes mencionan una frecuencia ligeramente mayor a favor del sexo femenino (1).

Esta lesión raramente ocurre en niños menores de 16 años (9). Generalmente es asintomática, causa expansión lenta del maxilar, que en raras ocasiones puede ser dolorosa $(3,10)$. Dos tercios de estos tumores afectan la mandíbula y un tercio la maxila siendo la localización más frecuente la región posterior (67\%) $(1,11)$. Algunos autores reportan igual incidencia en maxilar y mandíbula $(12,13)$. Considerando la localización el caso reportado este dato coincide con la literatura.

Desde el punto de vista radiográfico se ha descrito al mixoma con varios patrones: a) radiolucidez multilocular, cuyos márgenes pueden o no aparecer definidos (3), b) lesión con aspecto de pompas de jabón, c) imagen de raqueta de tenis, d) patrón en panal de abejas, e) lesión uniquística, f) lesión radiolúcida pericoronal (menos frecuente) y g) lesión mixta radiolúcidaradiopaca (raro) $(3,4)$. La presencia de radiopacidad puede deberse al hueso residual (14).

Algunas lesiones tienen gran extensión y comprometen completamente la rama mandibular o una gran 


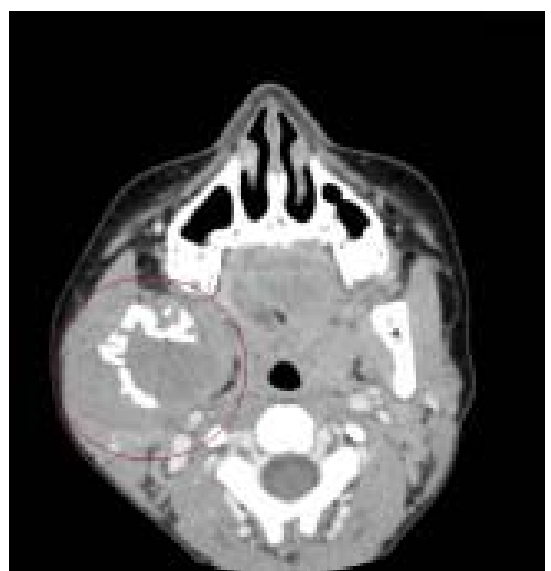

Fig. 8. TAC con sustancia de contraste: la lesión desplaza los músculos masetero y pterigoideos.

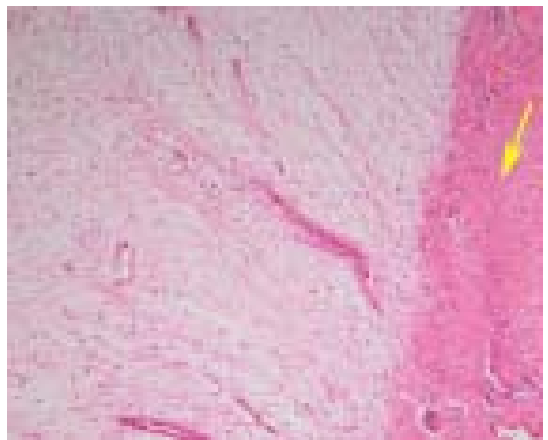

Fig. 10. Tejido fibromixomatoso con áreas de fibras colágenas (flecha). H\&E-10X.

extensión de la maxila (4). En el diagnóstico radiográfico diferencial deben considerarse radiolucideces multiloculares como el ameloblastoma, hemangioma central y granuloma central de células gigantes, entre otros $(2,7)$. Langlais et al. (15) han reportado algunos casos con apariencia de rayos de sol.

En nuestro caso el aspecto más relevante fue la imagen de "rayos de sol", que es frecuentemente atribuida a neoplasias malignas osteogénicas, específicamente al osteosarcoma, el cual se caracteriza por destruir las corticales óseas e infiltrar los tejidos circundantes al mismo tiempo que va produciendo tejido óseo sarcomatoso. Su crecimiento es rápido y está asociado a sintomatología neurológica, como dolor y parestesia. La invasión de partes blandas y márgenes indefini-

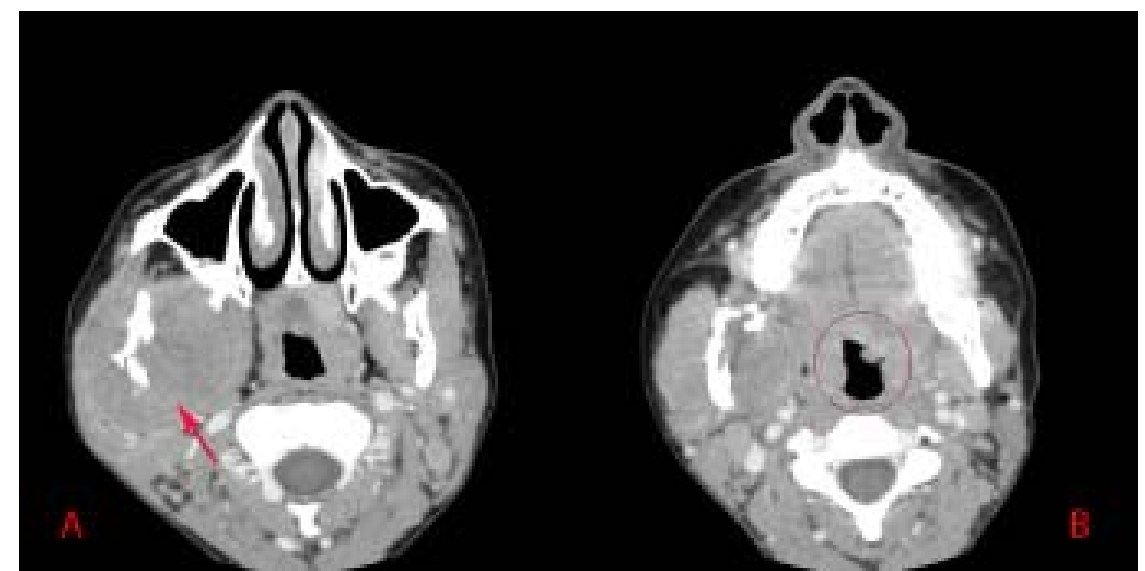

Fig. 9. TAC con sustancia de contraste: la lesión tumoral ha desplazado el músculo pterigoideo interno y los tejidos adyacentes produciendo estrechamiento del espacio orofaríngeo.

dos son considerados como criterios de malignidad (17).

El mixoma o fibromixoma odontogénico si bien es una lesión benigna es conveniente señalar que al examen histológico no muestra la cápsula fibrosa que caracteriza a los tumores benignos y más bien se la describe como una proliferación de células fusiformes y de forma estrellada dentro de una matriz mixomatosa con gran cantidad de fibras colágenas y trabéculas de hueso que producen compartimientos dentro del mismo tejido.

Debido a la ausencia de una cápsula fibrosa es que ocasionalmente puede destruir el hueso cortical e infiltrar los tejidos blandos adyacentes dando imágenes radiográficas con márgenes poco definidos y aparente invasión de partes blandas y presencia de tabiques óseos que dan imágenes de rayos de sol que conducen a confundirla con procesos malignos $(1,15,16)$. Esta característica histológica también explica la alta recidiva que presentan estas lesiones cuando no se plantean tratamientos quirúrgicos adecuados basados en el comportamiento biológico de esta lesión benigna.

En ocasiones, la radiología convencional no es suficiente para establecer en detalle las característi- cas del compromiso óseo y su extensión a los tejidos blandos, por lo que es fundamental recurrir a la tomografía espiral multicorte con y sin la administración de sustancia de contraste endovenoso. Con estos estudios se logran mejores imágenes para identificar los criterios de malignidad, el compromiso de tejidos blandos y la presencia de adenopatías $(17,18)$.

En nuestro caso, estos modernos estudios imaginológicos permitieron verificar que a pesar que el tumor estaba en contacto con las estructuras circundantes a través de las perforaciones de la tablas óseas, existía una delicada interfase que permitía separar el tumor de los tejidos blandos adyacentes, por lo que se pudo concluir que los tejidos normales estaban de alguna forma comprometidos pero no infiltrados (Figs.5-9).

En el mixoma, el aspecto en raqueta de tenis dado por la presencia de septos o tabiques óseos rectos intralesionales, pueden acompañar a la masa tumoral, las corticales pueden ser adelgazadas hasta el punto de no ser visibles a la inspección con radiología convencional, también pueden ser perforadas, y los septos pueden extenderse mas allá del margen óseo periférico en ángulos rec- 
tos, dando apariencia de rayos de sol $(2,15)$, lo que no debe ser confundido con reacción perióstica, como se observa en el osteosarcoma. La cortical también puede estar intacta pero adelgazada y expandida, permitiendo la clara identificación de los bordes lesionales (19).

Koseki et al. (19) usando tomografía computarizada para la evaluación de mixomas, señalan que los tabiques internos no son visibles en todos los casos y que cuando se observan no separan completamente la lesión en múltiples compartimientos. Cuando estos compartimientos están presentes, en la mitad de los casos exhiben el patrón de raqueta de tenis y los tabiques se observan generalmente en la periferia y no en el centro de la lesión, como se ha podido observar en el presente caso.

Algunos autores señalan que en ocasiones, el mixoma es indistinguible del osteosarcoma mediante técnicas de imagen (20), por lo que la presunción diagnóstica de benignidad o malignidad debe estar fundamentada en criterios clínicos integrados al análisis imaginológico ya que el pronóstico y el tratamiento serán diferentes (21). La literatura reporta casos sugestivos de malignidad por este "aspecto de rayos de sol" (22), así como también, casos de combinación de ambas entidades, los llamados mixosarcomas, que tienen mal pronóstico.

En cuanto al tratamiento, se recomienda la resección en bloque con márgenes de 1,5 a $2 \mathrm{~cm}$. de hueso sano (23), aunque no siempre esto es posible debido a la proximidad de estructuras vitales (24). Un gran número de pacientes son tratados mediante enucleación y legrado, pero las recidivas son bastante frecuentes habiéndose señalado hasta en un 25\% de los casos (7). En ge- neral el pronóstico suele ser bueno, cuando la resección del tumor es completa $(23,25)$.

El presente reporte de caso es un ejemplo del mimetismo que puede producir un tumor benigno en un estudio radiológico convencional, al presentar imágenes con características de tumor maligno. En nuestro caso la evaluación mediante el uso de una técnica de imagen complementaria como la tomografía computarizada, conjuntamente con una buena correlación clínica, fue fundamental para diferenciar dos lesiones de diferente comportamiento biológico como son el osteosarcoma y el mixoma o fibromixoma odontogénico.

\section{Referencias bibliográficas}

1. Barnes L, Eveson JW, Reichart PA, Sidransky D, editors. Pathology and genetics of head and neck tumours. Lyon: IARC Press; 2005.

2. Paniagua Chacón C, Calderón Ubaqui V, Beltrán Silva J. Un caso raro de fibromixoma odontogénico con apariencia radiográfica de "rayos de sol". Reporte de caso y revisión de la literatura. Rev Odontol Univ Sao Paulo 2008; 20(1):93-9.

3. Peltola J, Magnusson B, Happonen RP, Borrman H. Odontogenic myxoma--a radiographic study of 21 tumours. Br J Oral Maxillofac Surg. 1994; 32(5):298-302.

4. Piattelli A, Scarano A, Antinori A, Trisi P. Odontogenic myxoma of the mandible. Report of a case and review of the literature. Acta Stomatol Belg. 1994; 91(2):10110.

5. Chuchurru JA, Luberti R, Cornicelli JC, Dominguez FV. Myxoma of the mandible with unusual radiographic appearance. J Oral Maxillofac Surg. 1985; 43(12):987-90.

6. Cohen MA, Hertzanu Y. Myxofibroma of the maxilla: a case report with computed tomogram findings. Oral Surg Oral Med Oral Pathol. 1986; 61(2):142-5.

7. Wood NK, Goaz PW, Kallal RH. Odontogenic myoma. In: Wood NK, Goaz PW. Differential diagnosis of oral lesions. 4th ed. St. Louis: Mosby Year Book; 1991. P. 415-7.

8. Buchner A, Merrell PW, Carpenter WM. Relative frequency of central odontogenic tumors: a study of 1,088 cases from Northern California and comparison to studies from other parts of the world. J Oral Maxillofac Surg. 2006; 64(9):1343-52.

9. Ang HK, Ramani P, Michaels L. Myxoma of the maxillary antrum in children. Histopathology. 1993; 23(4):361-5.

10. Harder F. Myoxomas of the jaws. Int J Oral Surg. 1978; 7(3):148-55.

11. Olgac V, Koseoglu BG, Aksakalli N. Odontogenic tumours in Istanbul: 527 cases. Br J Oral Maxillofac Surg. 2006; 44(5):386-8.

12.Keszler A, Dominguez FV, Giannunzio G. Myxoma in childhood: an analysis of 10 cases. J Oral Maxillofac Surg. 1995; 53(5):518-21.

13. Regezi JA, Kerr DA, Courtney RM. Odontogenic tumors: analysis of 706 cases. J Oral Surg. 1978; 36(10):771-8.

14. Farman AJ, Nortjé CJ, Wood LE. Oral and maxillofacial diagnostic imaging. ST Louis: CV Mosby; 1993.

15.Langlais R, Langland O, Nortjé C. Diagnostic imaging of the 
jaws. EEUU: Williams \& Wilkins; 1995.

16. Moshiri S, Oda D, Worthington P, Myall R. Odontogenic myxoma: histochemical and ultrastructural study. J Oral Pathol Med. 1992; 21(9):401-3.

17.Kurabayashi T, Ida M, Tetsumura A, Ohbayashi N, Yasumoto M, Sasaki T. MR imaging of benign and malignant lesions in the buccal space. Dentomaxillofac Radiol. 2002; 31(6):344-9.

18. Sumi Y, Miyaishi O, Ito K, Ueda $\mathrm{M}$. Magnetic resonance imaging of myxoma in the mandible: a case report. Oral Surg Oral Med Oral Pathol Oral Radiol Endod. 2000; 90(5):671-6.

19.Koseki T, Kobayashi K,
Hashimoto K, Ariji Y, Tsuchimochi M, Toyama M, Araki M, Igarashi C, Koseki Y, Ariji E. Computed tomography of odontogenic myxoma. Dentomaxillofac Radiol. 2003; 32(3):160-5.

20. White S, Pharoah M. Radiología Oral Principios e interpretación. 5ta. ed. Madrid: Mosby; 2004.

21.Zhang J, Wang H, He X, Niu Y, Li X. Radiographic examination of 41 cases of odontogenic myxomas on the basis of conventional radiographs. Dentomaxillofac Radiol. 2007; 36(3):160-7.

22.de Labrouhe C, Bertand JC, Guilbert F. [Radiologic aspects of myxoma of the jaws. A series of 27 cases] [Article in French].
Rev Stomatol Chir Maxillofac. 1994; 95(2):80-3.

23. Halfpenny W, Verey A, Bardsley $\mathrm{V}$. Myxoma of the mandibular condyle. A case report and review of the literature. Oral Surg Oral Med Oral Pathol Oral Radiol Endod. 2000; 90(3):348-53.

24. Tincani AJ, Araújo PP, DelNegro A, Altemani A, Martins AS. Childhood maxillary myxoma: case report and review of management. Oral Surg Oral Med Oral Pathol Oral Radiol Endod. 2007; 104(5):e5-8.

25.Landa LE, Hedrick MH, Nepomuceno-Perez MC, Sotereanos GC. Recurrent myxoma of the zygoma: a case report. J Oral Maxillofac Surg. 2002; 60(6):704-8. 